\title{
Towards Standardising SHPB Testing - A Round Robin Exercise
}

\author{
Reuben Govender ${ }^{1, *}$, Muhammad Kariem $^{2}$, Dong Ruan ${ }^{3}$, Rafael Santiago ${ }^{4}$, Dong Wei Shu ${ }^{5}$, Marcilio Alves ${ }^{6}$, Guoxing \\ $\mathrm{Lu}^{6}$, Gerald Nurick ${ }^{1}$, and Genevieve Langdon ${ }^{1}$ \\ ${ }^{1}$ Blast Impact and Survivability Research Unit, Department of Mechanical Engineering, University of Cape Town, Private Bag X3, \\ Rondebosch, 7701, South Africa \\ ${ }^{2}$ Bandung Institute of Technology, Bandung, West Java 40132, Indonesia \\ ${ }^{3}$ Swinburne University of Technology, PO BOX 218, Hawthorn, Victoria 3122, Australia \\ ${ }^{4}$ Federal University of ABC (UFABC), 09210-580, Brazil \\ ${ }^{5}$ Nanyang Technological University, 50 Nanyang Avenue, 639798, Singapore \\ ${ }^{6}$ University of São Paulo (USP), Butantã, São Paulo - State of São Paulo, 03178-200, Brazil
}

\begin{abstract}
The Split Hopkinson Pressure Bar (SHPB) test, while widely utilised for high strain rate tests, has yet to be standardised. As an exploratory step towards developing a standard test method or protocol, a Round Robin test series has been conducted between four institutions: (i) Swinburne University of Technology, Australia (ii) University of São Paulo, Brazil, (iii) University of Cape Town, South African and (iv) Nanyang Technological University, Singapore. Each institution prepared specimens from a metallic material, and provided batches of specimens from their chosen material to the other institutions. The materials utilised in this round of testing were commercially pure copper and aluminium, magnesium alloy and stainless steel ( 316 grade). The intent of the first exercise is to establish the consistency of SHPB test results on nominally identical specimens at comparable elevated strain rates, conducted by different laboratories following notionally similar test procedures with some freedom in data processing. This paper presents and compares the results of the first batch of tests for copper, identifying variations between results from different laboratories. The variation between different laboratories' results for copper is sufficiently small that there is confidence in the potential to develop a draft standard in future.
\end{abstract}

\section{Introduction}

The Split Hopkinson Pressure Bar (SHPB) [1] or Kolsky Bar [2] test is a well established means of investigating mechanical response of a material to high strain rate $\left(\approx 10^{2}\right.$ to $\left.10^{4} \mathrm{~s}^{-1}\right)$ loading. The fundamental principles and guidelines for setting up, conducting and analysing a SHPB experiment are well described by Gray III [3], and Chen and Song [4]. However, conducting a successful SHPB experiment requires decisions on a large range of parameters (e.g. bar materials, length, diameter, specimen dimensions etc) and post-processing methods (1,2 or 3 wave analysis, dispersive or non-dispersive wave propagation etc). No standard describes reasonable starting values for these parameters for typical engineering materials.

To the best of the authors' knowledge, no data has been published showing that SHPB testing conducted at different laboratories, on specimens of the same provenance, produce consistent stress-strain results to within an acceptable tolerance. This article documents a Round Robin test series, which sought to establish whether specimens of the same material, tested in compression at SHPB facilities at different institutions, would produce similar stress-strain results. The intent is to ascertain how much the raw experimental data varies, given different physical SHPB set

\footnotetext{
*e-mail: reuben.govender@uct.ac.za
}

ups, and how different data processing methods affect the stress-strain curves which are the ultimate output of the experiments. This would provide guidance for drafting a testing standard in future. Four institutions participated in the exercise, with each institution providing specimens of a nominated metallic material, prepared from a single source. Each institution performed SHPB tests on a batch of its own material, as well as three other batches of different materials, provided by the other institutions. The participating institutions, as well as the material provided, are detailed in Table 1.

\section{Experimental Details}

\subsection{Specimen Details}

Each laboratory manufactured 40 cylindrical compression specimens, of one of the materials specified in Table 1. The specimens were $6.0 \pm 0.1 \mathrm{~mm}$ in diameter and $4.0 \pm 0.1 \mathrm{~mm}$ in length. The end faces were machined parallel and polished using 600 grit SiC polishing paper. The diameter and length had tolerances of $\pm 0.1 \mathrm{~mm}$. Each laboratory retained 10 specimens for in-house testing, and shipped batches of 10 specimens to the three other institutions for testing. 
Table 1. Participants and materials.

\begin{tabular}{lll}
\hline Institution & Country & Material \\
\hline Nanyang Technological University (NTU) & Singapore & Magnesium AZ31B \\
Swinburne University of Technology (SUT) & Australia & Stainless Steel 316 \\
University of Cape Town (UCT) & South Africa & Aluminium 1100 \\
University of São Paulo (USP) & Brazil & Copper (commercially pure) \\
\hline
\end{tabular}

\subsection{Physical SHPB Details}

All of the laboratories used conventional compression SHPB arrangements, as shown in Figure 1, with the striker being driven by a gas gun. The participating laboratories used SHPB of different dimensions and materials, detailed in Tables $2 \& 3$.

Each laboratory aligned and calibrated their SHPB according to their preferred procedures (not described for brevity). Alignment was not measured, though bar misalignment can introduce errors [5] and measurement thereof is being considered for a follow up study. The target for each laboratory was a plastic strain of approximately $20 \%$, in a strain rate range of 1000 to $2000 \mathrm{~s}^{-1}$. Each laboratory varied striker velocity to achieve this target. However, it was not specified as to whether the strain rate range, or the maximum plastic strain, was the more important target. To avoid increasing experimental complexity and cost, relatively simple pulse smoothing / shaping methods were used: 4 layers of paper (NTU), 4 layers paper masking tape (UCT), 3 layers of paper (SUT), while USP did not employ any pulse smoothing or shaping. Specimens were positioned by hand and lubricated with: common grease (NTU \& SUT) petroleum jelly (UCT), while USP did not apply lubricant.

\subsection{Instrumentation details}

All the laboratories used a pair of diametrically opposed foil resistance strain gauges (nominal resistance $120 \Omega$ ) on each bar. The positions of the gauges on the input and output bar are noted in Table 2, while details of the amplification and data acquisition are provided in Table 4.

\subsection{Data Processing}

The data processing methodology was not prescribed as part of the exercise, to help ascertain how much variation in final results the different methods would induce. In this exercise, all laboratories employed input and output bars of equal diameter $d$, and therefore the area of the input and output bars $A_{B}$ are equal. Obtaining specimen stressstrain from an SHPB experiment requires several decisions about how to process the captured incident $\varepsilon_{I}$, reflected $\varepsilon_{R}$ and transmitted $\varepsilon_{T}$ strain waves. Specimen strain may be calculated purely based on $\varepsilon_{R}$ (1-Wave method), which assumes specimen equilibrium, or using all of $\varepsilon_{I}, \varepsilon_{R}$ and $\varepsilon_{T}$ (3-Wave method), which does not inherently assume

${ }^{1}$ Gain is constant to $100 \mathrm{kHz}$, gain drop off and phase shift characterised up to $1 \mathrm{MHz}$ and corrected in post processing equilibrium [3]. Similarly specimen stress may be calculated from only $\varepsilon_{T}$ (1-Wave), superimposing $\varepsilon_{I}$ and $\varepsilon_{R}$ (2Wave), or averaging input and output face forces, which uses $\varepsilon_{I}, \varepsilon_{R}$ and $\varepsilon_{T}$ (3-Wave). All these methods require that the wave captured at the strain gauge be shifted to the relevant bar-specimen interface. This shift can either assume 1-dimensional wave propagation, and hence no shape change; or it can correct for dispersion of the wave in the frequency domain using a phase shift calculated from the Pochammer-Chree phase velocity-frequency relationship (originally proposed in $[6,7]$, current procedure described in [8]). The identification of the start of specimen loading may be either by inspection, or using techniques such as proposed by Zhao and Gary [9] or Li and Lambros [10]. While each laboratory used different methods for calibrating the bars' wave speed $C_{o}$ and calibration factors for converting strain gauge voltage to actual strain, the details of these methods have been omitted from this publication for brevity. All laboratories confirmed dynamic equilibrium of the specimen for the test to be acceptable. The different processing methods employed by each laboratory are summarised in Table 5.

\section{Results and Discussion}

While four different materials have been tested in this exercise, only the data for copper are presented in this paper due to space constraints. Before data from any test was considered valid, the equilibrium of forces on the input and output faces of the specimen was checked. Some examples of this for each laboratory are shown in Figure 2. The valid data sets from each laboratory for copper are shown in Figure 3. The maximum plastic strain varies between laboratories, as is expected due to the different striker lengths and velocities. The strain rates stated in each figure are the average strain rate, taken from the peak rate to the point where the specimen begins to unload. As each laboratory could vary striker velocity, there is a variation in strain rates achieved between laboratories.

As is usual for SHPB data, there are oscillations on any given specimen's stress-strain curve as well as the usual specimen-to-specimen variation. Attempting to overlay all the data from the subfigures of Figure 3 on a single figure makes it very difficult to distinguish one batch of data from another. A reduced set of points from all of the flow stress curves from each laboratory are shown overlaid in Figure 4a. It is clear that there is significant overlap in the data from each laboratory. To help discern differences between the four laboratories' data sets, the entire flow stress data set from a single laboratory is fit to a power law, $A+B \varepsilon^{n}$. 


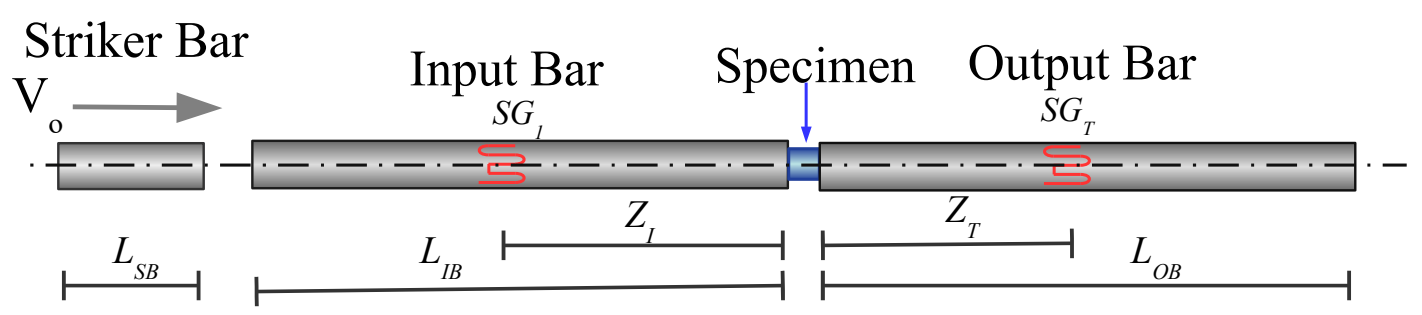

Fig. 1. Schematic of SHPB.

\begin{tabular}{lcccc}
\multicolumn{5}{c}{ Table 2. SHPB Dimensions. } \\
\hline Parameter & NTU & SUT & UCT & USP \\
\hline Bar Diameter $D_{B}(\mathrm{~mm})$ & 12.7 & 14.5 & 12.7 & 24.99 \\
Striker Length $L_{S B}(\mathrm{~mm})$ & 300 & 300 & 350 & 253 \\
Input Bar Length $L_{I B}(\mathrm{~mm})$ & 1000 & 1200 & 1199 & 1990 \\
Output Bar Length $L_{O B}(\mathrm{~mm})$ & 1000 & 1200 & 700 & 1990 \\
$S G_{I}$ to specimen $Z_{I}(\mathrm{~mm})$ & 500 & 500 & 599.5 & 1000 \\
Specimen to $S G_{T} Z_{T}(\mathrm{~mm})$ & 501 & 400 & 100 & 1000 \\
\hline
\end{tabular}

Table 3. SHPB Mechanical Properties.

\begin{tabular}{|c|c|c|c|c|}
\hline Parameter & NTU & SUT & UCT & USP \\
\hline \multirow{2}{*}{ Bar Material } & Stainless & Maraging & Stainless & Steel SAE \\
\hline & Steel 304 & Steel 350 & Steel 17-4 PH & 1055 \\
\hline Density $\rho\left(k g . m^{-3}\right)$ & 8000 & 8070 & 7735 & 7816 \\
\hline Wave Speed $C_{o}\left(m \cdot s^{-1}\right)$ & 5138 & 4800 & 5025 & 5082 \\
\hline Elastic Modulus $E(\mathrm{GPa})$ & 200 & 186 & 195 & 194 \\
\hline Poisson Ratio $v$ & - & 0.31 & 0.3 & 0.29 \\
\hline \multicolumn{5}{|c|}{ Table 4. Instrumentation details. } \\
\hline Parameter & NTU & SUT & UCT & USP \\
\hline Amplifier Gain & 1000 & 300 & 1000 & $\mathrm{n} / \mathrm{a}$ \\
\hline Upper Frequency Limit $(\mathrm{kHz})$ & 3000 & 500 & $1000^{1}$ & 500 \\
\hline Bridge Excitation $V_{B R}(V)$ & 2.46 & 2 & 2.27 & 5 \\
\hline DAQ Resolution (bits) & 8 & $\mathrm{n} / \mathrm{a}$ & 16 & 12 \\
\hline DAQ Full Scale $(V)$ & 0.5 & 0.5 & \pm 5 & $\mathrm{n} / \mathrm{a}$ \\
\hline Sampling Rate $\left(M S a . s^{-1}\right)$ & 1.25 & 5 & 10 & 5 \\
\hline Sampling Duration $(m s)$ & 1.0 & 0.5 & 1.5 & 80 \\
\hline
\end{tabular}

Table 5. Data processing methodologies.

\begin{tabular}{lcccc}
\hline & NTU & SUT & UCT & USP \\
\hline Wave Shifting & 1-D & 1-D & Disp. Corr. & 1-D \\
Specimen Strain Calc. & 1-Wave & 1-Wave & 3-Wave & 1-Wave \\
Specimen Stress Calc. & 1-Wave & 1-Wave & 1-Wave & 1-Wave \\
Start of loading & Insp. & Insp. & Insp. & Insp. \\
\hline
\end{tabular}

An example of the power law fit to all specimen plastic flow data for a single laboratory is shown in Figure $4 \mathrm{~b}$.

Figure 5 shows the power law flow curves fit to each laboratory's data set for copper, which makes some subtle differences more obvious. Based on the power laws, the average flow stress at $\varepsilon=5 \%$ is $335 \mathrm{MPa}$, with a standard deviation of $7 \mathrm{MPa}$. As the strain increases to $20 \%$, the average flow stress increases to $377 \mathrm{MPa}$ while the standard deviation also increases to $11 \mathrm{MPa}$. In the ASTM Standard Test Method for Quasi-Static compression of metals at room temperature [11], the $95 \%$ reproducibility limit for Al-2024 with a yield strength of
$346 \mathrm{MPa}$ was $19 \mathrm{MPa}$ between laboratories. This gives good confidence in the reproducibility between laboratories conducting SHPB tests at high strain rates. The flow stress for the SUT data is higher than the other laboratories, though marginally so than that of USP. The flow stresses from the UCT and NTU data are practically indistinguishable. The SUT tests were conducted at the highest average strain rate $\dot{\varepsilon} \approx 2.55 \times 10^{3} \pm 0.12 \times 10^{3} \mathrm{~s}^{-1}$. The UCT tests were conducted at the lowest average strain rate $\dot{\varepsilon} \approx 1.05 \times 10^{3} \pm 0.12 \times 10^{3} s^{-1}$. The strain rate bands for NTU and USP overlap, at $\dot{\varepsilon} \approx 1.6 \times 10^{3} \pm 0.3 \times 10^{3} s^{-1}$ and $\dot{\varepsilon} \approx 1.5 \times 10^{3} \pm 0.1 \times 10^{3} s^{-1}$ respectively. If the variation 


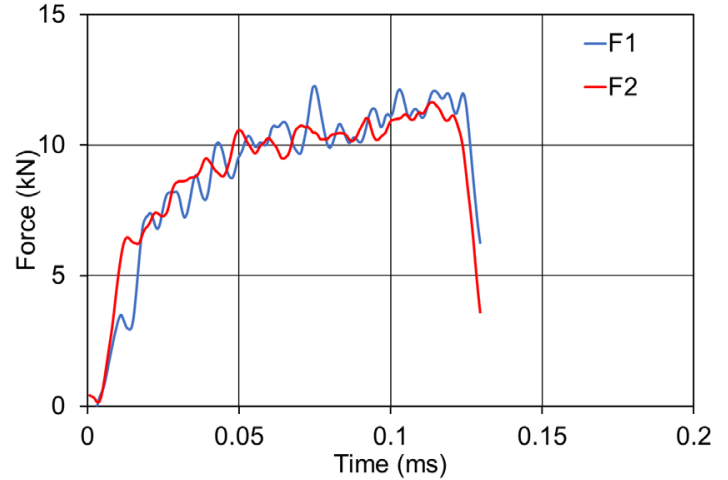

(a) NTU

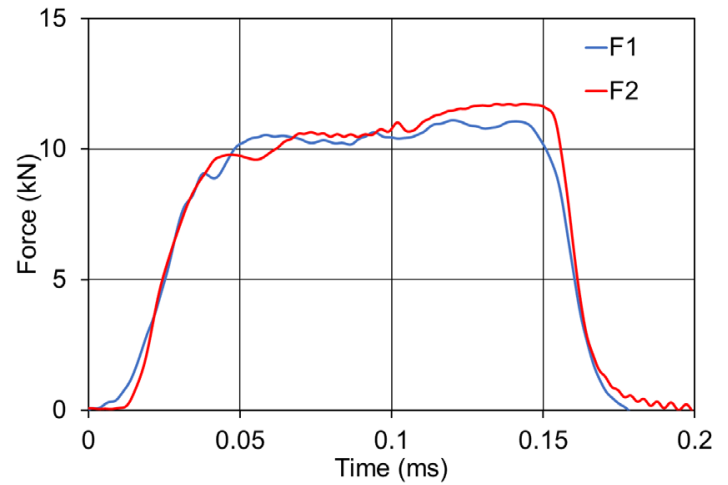

(c) UCT

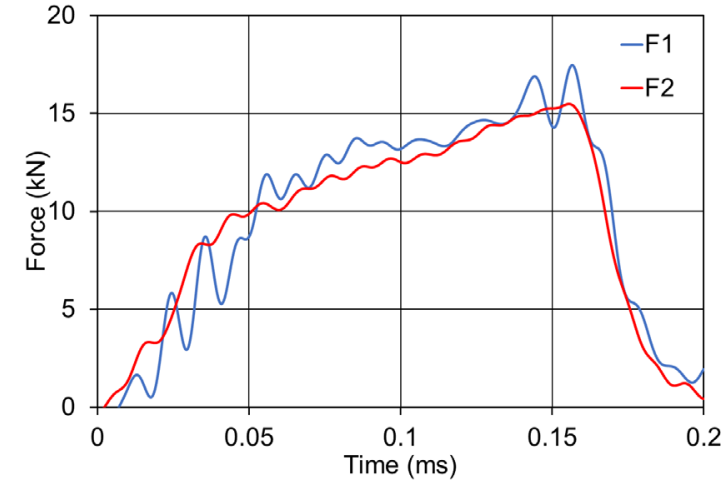

(b) SUT

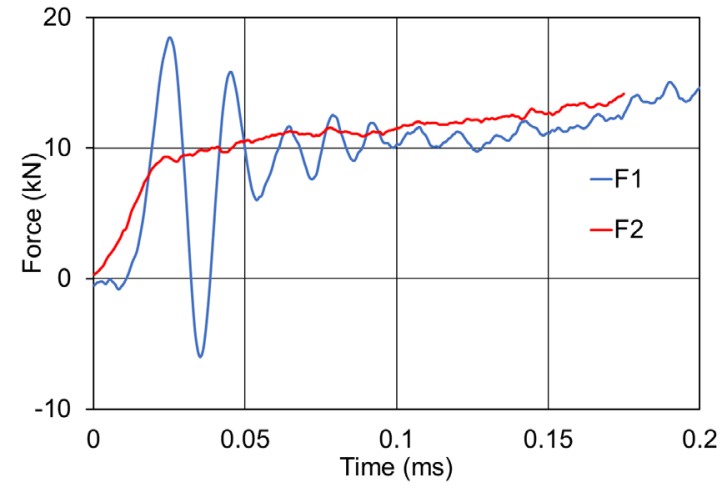

(d) USP

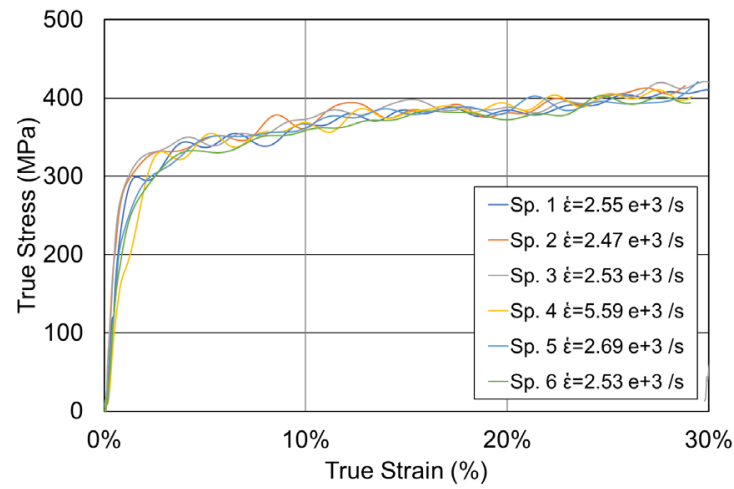

(b) SUT

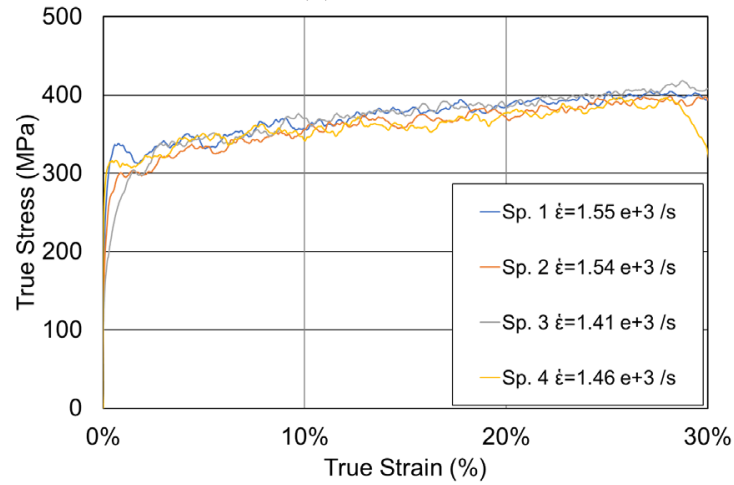

(d) USP

Fig. 3. True stress-true strain data for valid copper tests from all laboratories. 


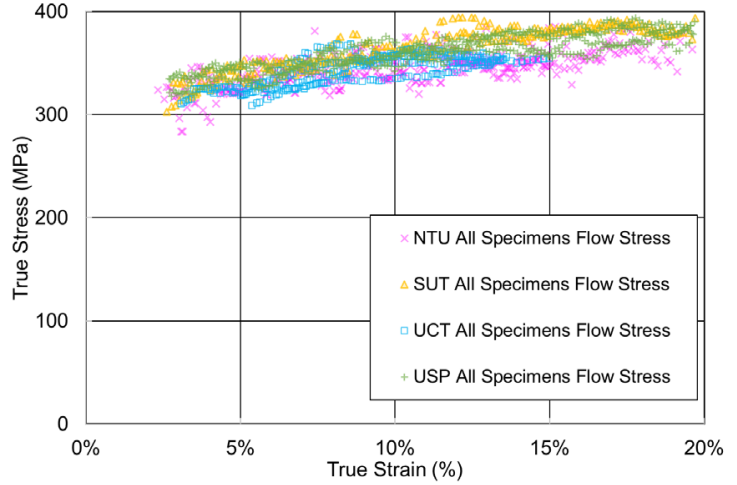

(a) Flow stress point cloud for all valid copper tests from all laboratories.

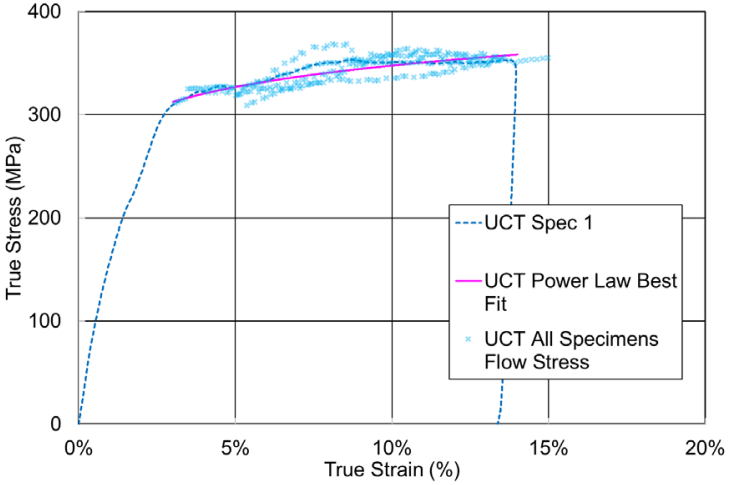

(b) Power law fit to all UCT data for copper.

Fig. 4. Flow stress data for copper and power law fitting.

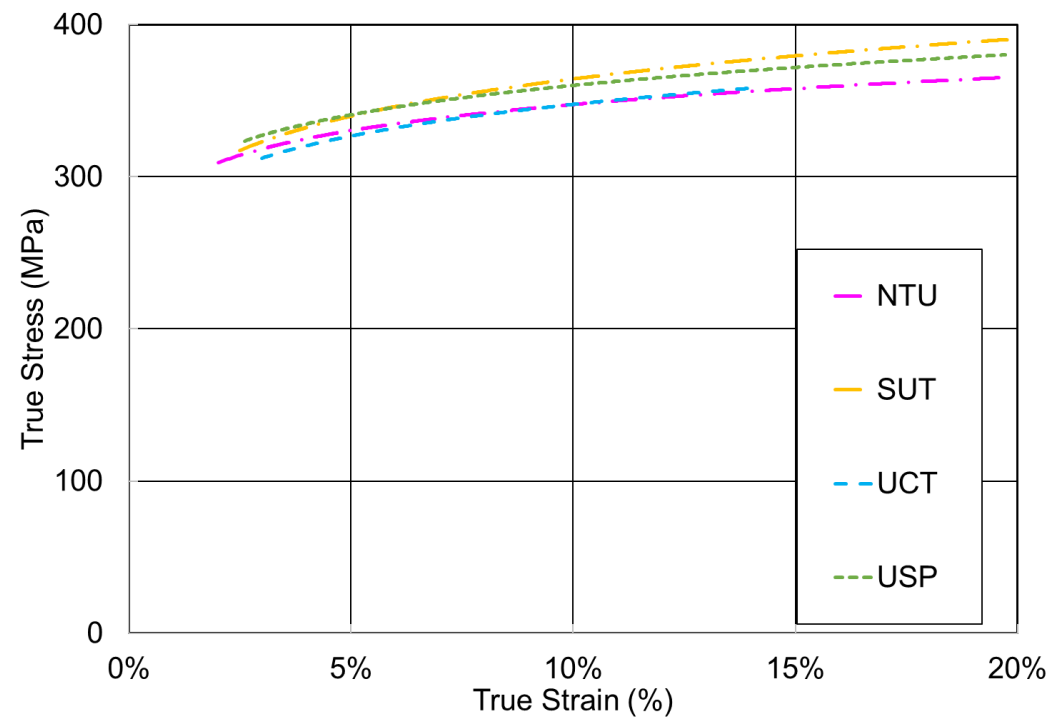

Fig. 5. Best fit power law flow curves for all laboratories for copper specimens.

between the laboratories was purely due to strain rate, the data from NTU and USP should coincide, which is not the case. An analysis of the variations due to data processing methods and other experimental variables is not possible in this article due to space constraints. Nonetheless, the variation between laboratories' data sets is small enough that there is confidence moving forward.

\section{Concluding Remarks}

Four laboratories participated in a Round Robin exercise aimed at developing a standard test method for SHPB testing of metallic materials. The laboratories all used different physical SHPB arrangements, as well as instrumentation and data processing. The variation between flow stress curves shows that different laboratories can produce consistent data for the same specimens, even though a relatively broad range of instruments and data processing methods was employed. This suggests that a future standard test method for SHPB tests need not be onerously prescriptive in order to obtain consistent results. More detailed analysis of the variations between laboratories in experimental apparatus, test method and data analysis is planned for future publications which are less space constrained. It is hoped that a further experimental Round Robin series can be conducted with more participants and a more refined prescribed method.

\section{References}

1. R. Davies, Phil. Trans. Royal Soc. London Part A 240, 375 (1948)

2. H. Kolsky, Proc. Physical Soc. Section B 62, 676 (1949)

3. G. Gray III, Mechanical Testing and Evaluation (ASM International, 1999), Vol. 8 of ASM Handbook, chap. Classic Split-Hopkinson Pressure Bar Testing, pp. 462-476

4. W.W. Chen, B. Song, Split Hopkinson (Kolsky) bar: Design, Testing and Applications (Springer Science \& Business Media, 2010)

5. M.A. Kariem, J.H. Beynon, D. Ruan, International Journal of Impact Engineering 47, 60 (2012)

6. D. Gorham, Journal of Physics E: Scientific Instruments 16, 477 (1983) 
7. P. Follansbee, C. Frantz, Journal of Engineering Materials and Technology 105, 61 (1983)

8. S. Marais, R. Tait, T. Cloete, G. Nurick, Latin American Journal of Solids and Structures 1, 219 (2004)

9. H. Zhao, G. Gary, International Journal of Solids and Structures 33, 3363 (1996)
10. Z. Li, J. Lambros, Composites Science and Technology 59, 1097 (1999)

11. ASTM International, ASTM E9-09(2018) Standard Test Methods of Compression Testing of Metallic Materials at Room Temperature, ASTM International (2018) 